\title{
A Review Study of Heartbeat Biometric Authentication
}

\author{
Ala Abdulhakim Alariki ${ }^{1}$, Sayed Mahmoud Alavy ${ }^{1 *}$, Mohammad Reza Yousufi ${ }^{1}$, Mohammad \\ Tareq Aziz ${ }^{1}$, Christine Murray ${ }^{2}$ \\ ${ }^{1}$ Information Technology Department, American University of Afghanistan (AUAF), Kabul, Afghanistan. \\ 2 English and Humanities Department, American university of Afghanistan (AUAF), Kabul, Afghanistan. \\ * Corresponding author. Email: mahmoodalavi.af@gmail.com \\ Manuscript submitted December 10, 2017; accepted January 18, 2018. \\ doi: $10.17706 /$ jcp.13.8.936-947
}

\begin{abstract}
Today, life engages technology in multiple ways, thus authentication in human technologies is very important. Secure and reliable authentication is in high demand. However, traditional methods for authentication such as passwords and tokens are now outdated because it is possible to steal, lose and share such authentication methods. Current research shows that one of the best methods for authenticating human beings is biometrics. In this paper, the heartbeat biometric, also called Electrocardiographic (ECG), is proposed. The heartbeat biometric is chosen because unique human ECGs cannot be falsely created or replicated. While other biometric methods, such as face recognition, can be compromised by user photographs, or fingerprints, which can be compromised by use of fake fingers, the ECG signal is based on the individualized mechanical movements of each human heart, which features contain unique physiological information. The purpose of this paper is, then, to review various relevant, recent works that study the heartbeat biometric to find the best biometrics features, given the extractions and classification algorithms for the heartbeat biometric signal. This paper concludes that the morphological ( $\mathrm{P}$ wave) feature is recommended as the most important feature and the Neural Network (NN) classifier is the most reliable classification with the highest performance accuracy for heartbeat biometric. Therefore, to achieve highest accuracy and result for authenticating through heartbeat biometric, it is recommended to consider the mentioned feature extractions and classification.
\end{abstract}

Key words: Biometric, heartbeat recognition, authentication, morphological, morphological, ECG (T wave), ECG (QRS complex).

\section{Introduction}

Human authentication has become an important aspect of daily life. One of the reasons is the increasing use of technology to enhance human activity and lifestyle. In general, human authentication and/or unique recognition is the way humans prove unique use, or show use, of services by proving originality [1]. Passwords and tokens are common, traditional types of authentication, yet each also has disadvantages: passwords are easily guessed, thereby easily stolen, for example, simply through "shoulder surfing." Also, common types of password authentication generally provide inadequate security levels, which increase the risk of unauthorized access. These include widespread use of easily memorized, simple combinations, and the method of writing down passwords not easily memorized [2]. Furthermore, a token can easily be lost, causing difficulty in issuing multiple new passwords over time [3].

However, biometrics solved these password and token issues. Unlike passwords and tokens, the 
attributes of biometrics cannot be lost, destroyed, or stolen via physical access. Moreover, the features of biometrics are very difficult to hack because a genuine person must forge them [4]. For instance, many people currently use online applications for purchasing goods. To decrease the chance of hacking and fraud, an authentication system with high level security, such as biometric, is necessary. In addition, for increasing the limitation on physical access, companies mostly recommend using biometrics instead of passwords and tokens [4].

In all, biometric systems are based on anatomical, physiological, and behavioral characteristics that are mostly unique between individuals [5]. Anatomical features are based on representative body parts that are not easily observable with humans [3], such as the face or hand geometry [6]. Physiological biometrics work directly with measurement of human body parts, for example in ear recognition, fingerprint, palm print, iris recognition and heartbeat recognition [1]. Additionally, there are behavioral characteristics, the reflection of an individual's psychology [7].

While traditional biometrics are an improvement, they also have features and weaknesses that cause drawbacks; thus no single method is expected for use in all types of application [5]. The drawbacks of traditional biometrics, such as found in fingerprints, retina and human face recognition, are inconsistent performance, costliness, non-standardized, low degree of user acceptability, and intrusive for collectability [2].

Yet heartbeat biometric authentication can solve the current drawbacks of traditional biometrics because heartbeat biometrics work primarily with the heartbeat signal as detected from the living body. There are several advantages to heartbeat biometrics. First, it is very difficult to steal or replace the signal, because major challenges exist in obtaining or creating fake signals [8]. Secondly, integration, of high quality heartbeat signals for recognition is primarily collected from fingerprinting to thus create integration via two-type authentication. Thirdly, performance is an advantage because the common way of evaluation is the error rate of false accept (FAR) and false reject (FRR) [8].

The procedure for identifying the rudiments of the heart is to detect signals of the individual heartbeat through ECG devices. ECG signals are identified as based on heart sound signal and its power spectrum which determine the T-wave, P-wave and QRS-Complex of the ECG samples. It is then based on the frequency, time and peaks of the signal recorded as data. Each of the collected heartbeat signals in a time frame is called a specimen. This specimen is the contained sequence of the unique heartbeat [9].

This paper is organized as follows: in Section 2, the behavior of heartbeat wavelet-related works are given. In Section 3, heartbeat biometric framework is described. In Section 4, the performance measurement is discussed. Finally, conclusions and future work are shown in Section 5.

\section{Related Works}

According to our research on the exact methodology and features of heart wave biometrics, each study proposed the heart wave signal as a biometric authentication method. In fact, the differences of the methods in identification and the baseline of wavelet of heart signals on each of the researched technics are proposed in this work. Each of the recent related work researching ECG wave authentication technics and performance are briefly organized as follow:

Abo-Zahhad, Ahmed et al (2014) [1], proposed the multimode heart wave biometric authentication system based on Electrocardiogram (ECG) and Phonocardiograph (PCG). Their article describes ECG time (fiducial)-based and ECG frequency-based as unique features, using the Neural Network (NN) as a classification. In this research, they argue that heart electrical activity and its sound are highly desirable for applications such as human biometric authentication. In this case, they compared differing approaches for ECG and PCG biometric authentication in their article, stating the features of ECG biometric and their 
accuracy or result depend on the location of the sensor. They elaborate as to how the ECG signals can be archived in difficult situations or cardiac irregularities, such as PVC and APC. With their 20 research subjects, the frequency result at $1-50 \mathrm{~Hz}$ with the $80 \%$ rate of success shows the position of the sensor is not always in a stable position yet still they achieved an accuracy that attained positive results.

Alajlan, Islam et al (2013) [10], proposed the use of fuzzy adaptive genetic algorithm (FAGA) as a method for developing performance of fingerprint biometric system by integration with heartbeat. The evaluation of multimodal system on this research paper was done on a larger database. They used robust morphological features of the heartbeat wavelet from fingers in differing fusion rules with linear combination of matching scores on fingerprint and heartbeat modalities. Their records and tests has been done on 112 individuals with feature of Morphological (P Wave) and Fuzzy Logic classification. The author's findings are based on their experimental results with matching of two different sets of data. They pointed out that the output result indicates a significant margin error rate level reeducation by $2.38 \%$ and $1.79 \%$. The potential suggestion is helpful in terms of heartbeat capturing performance to improve security in future practical-applications.

Brás and Pinho (2015) [11], claimed the ECG signal delineation or segmentation generally has a low accuracy especially when the wave is affected by noise. As a result, they proposed alternative ways: nonfiducial and semi-non-fiducial methods with R-peak delineation of aligning. Thus, they mentioned that the ECG has rhythm and amplitude. Therefore, it requires a system to deal with the variability of wavelet and noise sources, attaining a conditional result. They came up with the ECG frequency-based as a feature to control and deal with the complexity of measurement of heartbeat signals. They continued by comparing similarity and dissimilarity measures with a method of information-theoretic data models for data solidity. Their database data was collected from 52 participants over differing days, and they took from each participant five segments of 20 seconds ECG data. To evaluate and identify the biometric performance they used a 1-NN (Nearest Neighbor) classifier to come up with $99.91 \%$ accuracy. The paper conclusion states that the data is collected in different days because of the result could be affected by external variability conditions, such as stress. In future study of authentication layers, this major condition should be considered and analyzed to discern the NCR value in an acceptable interval for that particular person.

Islam and Alajlan (2014) [12], also proposed a model-based alignment method for heartbeat morphology for enhancing recognition capability. They tested their recognition capability in two different databases of heartbeat morphology records to claim that the method of model-based alignment is valuable to boost the recognition capability of heartbeat morphology. Thus, they came up with the statistical t-test records showing improvement is important in respect of recognition capabilities given other existing alignment methods. Two different alignment methods are used to measure the intra-individual and inter-individual distances of aligned heartbeats. However, they used the three types of morphological features P-Wave, QRS complex and T-Wave with the Nearest Neighbor (NN) classification on 112 participants with 70.53\% accuracy. The signals have been acquisitioned from fingers using ECG Handheld device.

Li and Li (2014) [13] proposed an ECG verification biometric system for healthy and cardiac irregular conditions of humans using of two information fusions, heartbeat level and segment level. For normalization, they used the outlier removal algorithm after peak detection to have the result of extract normalized representative beats. However, they applied the principal component (PCA), linear discriminant analysis (LDA) and within-class covariance normalization (WCCN) to align the beat variability compensation with the similarity of cosine and Snorm as wavelet scoring. At this level, they unsupervised joint-segmentation-peak-detection with joint-segmentation and clustering with-out peak. Then, they claimed it will automatically decode each raw signal into a string victor at the end the applying of 3-gram 
language model with log likelihood ratio based on hypothesis testing system for collection of scoring. Therefore, on the process of research, they used the Morphological P-Wave as a feature with classification of Nearest Neighbor (NN) to identify their 290 participants with 2500 iterations sampling by usage of ECG device with accuracy level of $23.6 \%$ EER. They concluded that heartbeat and segment level fusion systems for the cross-session verification task have some exciting unseen out-of-set testing samples and cardiac irregular data and all these will come up with the overall system performance.

Nomura, Ishikawa et al (2014) [14], they came up with a personal authentication method to propose that the usage of heartbeat waveform that could enhance the security in wireless communication. They continued, that the human heartbeat waveforms have a special characteristic features that mimicking would be very hard. They used the chaos indicator of ECG for personal authentications method that their method provides better authentication than existing ones with help of correlation dimension and its intercept, RMS, and approximate entropy. Hence, their research on the heartbeat biometric authentication done with feature of the Chaos Indicator of ECG and with usage of two types of classifications such as Neural Network (NN) and SVM. They experiment the research on 20 participants with NN classification accuracy of $93.7 \%$ on 9 examinees and the accuracy has been decreased when the test done on 20 examinees to $81.0 \%$. The accuracy increased when the test done based on SVM classification by $86.5 \%$ on 20 participants. Their last suggestion is that the number of examinees and samples will have more effective index for a research and it requires financial support.

Pathoumvanh, Airphaiboon et al (2014) [5], they proposed to study two types of realistic application themes for ECG biometric identification, as counted as follow; the single beat of an electrocardiogram (SBECG) used as an ECG feature to decrease time to acquire the ECG from the individual. The other one is the robustness study of ECG features to heart rate variability (HRV). They did relation comparison between the HRV conditions with the correct identification. The method of Continuous Wavelet Transform (CWT) is used for analyzing of SB-ECG parts that point out as a main novelty of this research. They used the P-Wave, QRS complex and T-Wave of ECG as a feature of their research with the normalized Euclidean Distance that proposed as a person identification of classification. They claimed that their identification method accuracy is about $97 \%$ and it took less time to acquire.

Pouryayevali, Wahabi et al (2014) [15] proposed in their paper as follows; they stated the credits goes to the ECG database records of University of Toronto (UofTDB) where a record of 1012 individual ECG data is stored for evaluating the performance of ECG biometric methods. This UofTDB uses three central characteristics, which are: large individual records, varying body postures, physical exercise and acquisition over a long period with 5\% EER rate. The paper uses the Euclidean Distance as a classification to apply the Autocorrelation (AC) of ECG feature through applying a new algorithmic evaluation, where the authors claimed that their standards for ECG biometric systems could have an impact on the affected waveform factors that will increase the benchmark of the accuracy rate. Hence, it could be introduced as a new evaluation benchmark method for ECG biometric authentication procedure.

Ramli, Hooi et al (2016) [9], proposed that in their study they developed a portable ECG detection kit for the heartbeat data capturing, and it is a wearable bracelet heartbeat detection device for personal use. Hence, they used the wavelet transform algorithm as a software feature of the device for extraction technique to apply the ECG P-wave, QRS Complex and T-wave features with classification of Support Vector Machine (SVM). Consequently, both the software and hardware techniques combined in a heartbeat biometric bracelet device and it's been experimented on 50 individuals that achieved the result of $2.0069 \%$ EER. They claimed, that their system has an easier troubleshooting procedure with two plots of the output signals that identifying the front-end system, which the first plot is on Android platform phone that able to 
store data after analyzing and filtering signals as a ${ }^{*}$.dat file. The second plot is a LabView oscilloscope that has the ability to view the output signals from the heartbeat detection kit. However, after the mentioned two plots the signals will be de-noised with stated ECG features.

\section{Methodology}

In this study, the methodology framework of heartbeat biometric system is designed based on reviewing recent related work studies.

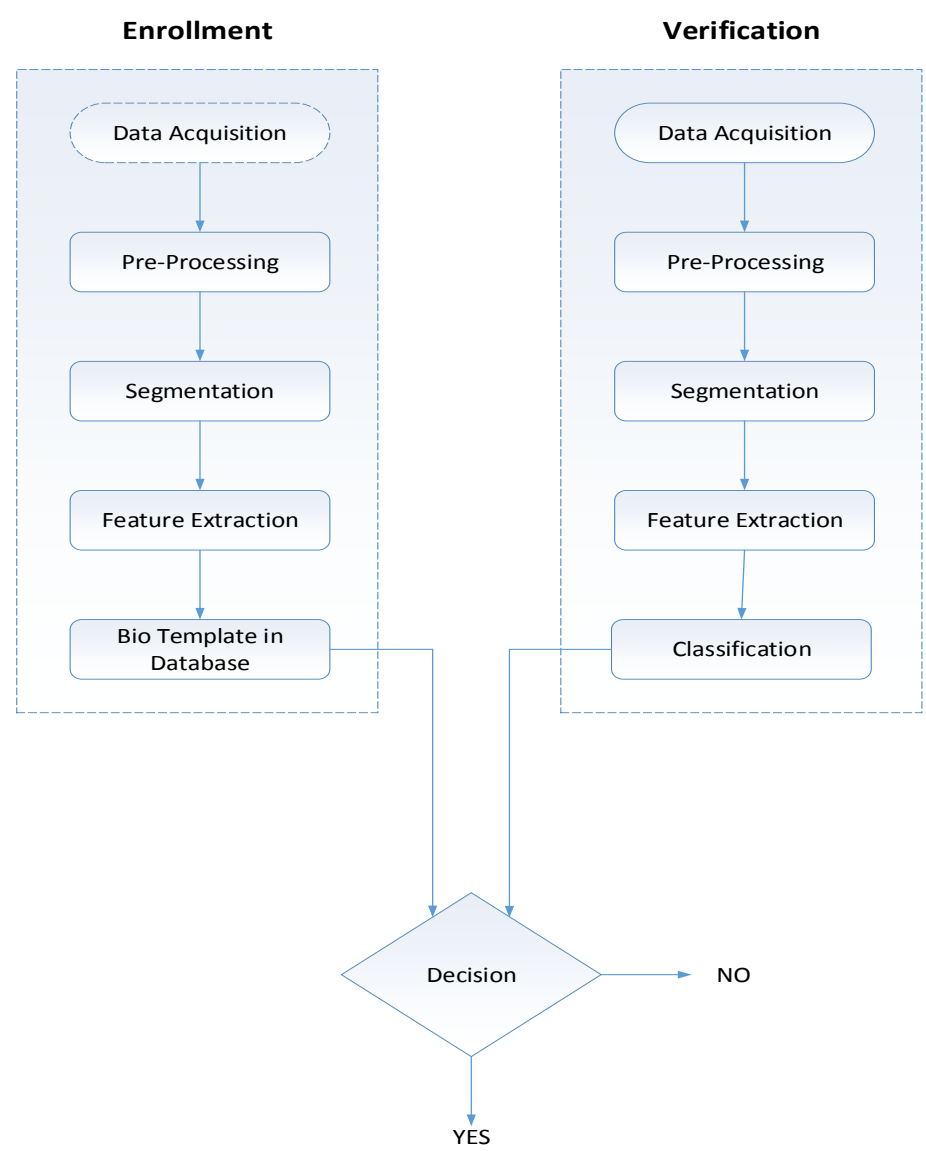

Fig. 1. Basic heartbeat biometric framework.

Fig. 1 shows basic framework of heartbeat biometric. The heartbeat biometric framework includes two phase as showing in Fig. 1. The first phase is storing the enrollment of each individual feature of heartbeat in a database. The second phase is verification that compares the acquisitioned data from individuals with the template in the database to find matches. Then the decision will be taken based on the comparison. Data Acquisition, Pre-Processing, Segmentation and Feature Extraction are the same steps in both enrollment and verification phases [1].

\subsection{Data Acquisition}

The first step is data acquisition or capturing. In this step a template extracts from inputted data. Different types of capturing used for heartbeat biometric in the reviewed related work studies. In one of them, heartbeat signals is captured from captured fingers from thumbs of both hands using two dry conducting electrodes. This capturing method is used for online users for authentication which is critical for improving security and privacy [16]. Another type of capturing is ECG capturing that captures with appropriate device like 2-lead palm scanner. In this type of capturing, ECG signal is captured in form of a 
scanned picture [7].

\subsection{Pre-processing}

The second step is pre-processing. In this step, there are several methods that are applied to signals for reduction and normalization. While ECG raw signal has noise, these signals should go through de-noise process before segmentation step. Therefore, after the pre-processing step, signals will be clear. Based on related studies, many methods have been used for pre-processing, in order to remove noises. One of the method for pre-processing is Wavelet de-noised process that suppress noise and observe signal pick clearly [9]. Another method of pre-processing for some noise such as power-line interface, baseline wanders and patient-electrode motion artifacts is band-pass Butterworth filter. In this method, Butterworth filter of order four with cut-off frequencies of 0.25 and $40 \mathrm{~Hz}$ is applied for heartbeat signal [16]. Moreover, there is another method for pre-processing which is called cepstrum. The method calculates the envelope of the measured data stream since noise of signals are generated frequently. Then the shape of envelope should be corrected to 0 and the noises will be removed using wavelet transform [14].

\subsection{Segmentation}

The third step is segmentation which detects peaks locations and boundaries of three types of waves, $\mathrm{P}$ wave, QRS complex, and T wave which are used in heartbeat signals. To evaluate the ECG signals, it's required to do mentioned features to identify the segment of a wavelet to be considered as a biometric identification method and it's been used in the papers that we researched on. Thus, ECG standard signals could be segmented with peaks boundaries and a classification method is to reach in an accurate result [17].

\subsection{Feature Extraction}

Feature extraction is a procedure to make a template from biometric data which is acquisitioned from user through recognizing and distinguishing properties. The purpose of the feature extraction is to have identical template from same user and different template from different users. This is one of the main step of biometric heart bear because in this step vector features will be extracted from heartbeat signals as input [2].

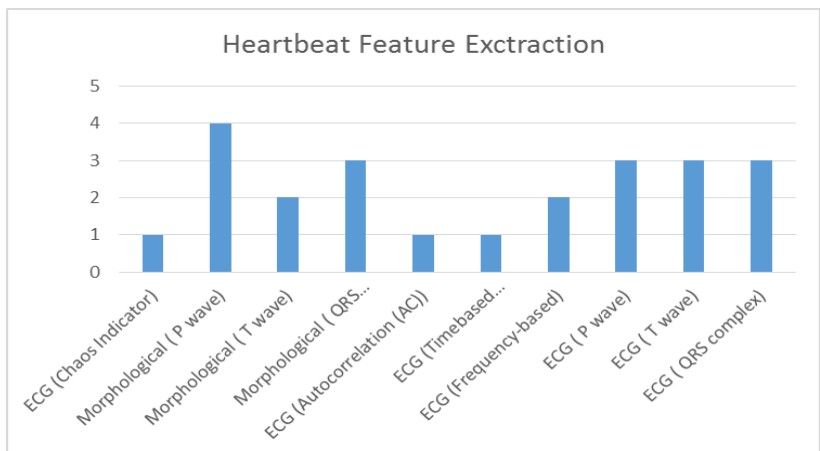

Fig. 2. Heartbeat feature extraction comparison.

Fig. 2 shows the heartbeat single features used by recent related work studies. The most significant feature used by the related work studies is Morphological ( $\mathrm{P}$ wave) feature. This feature used by four different studies which are Alajlan, Islam et al. 2013, Islam and Alajlan 2014, Li and Li 2014, Abo-Zahhad, Ahmed et al. 2014. As a result, it shows we can differentiate between individuals with more accurate result by using the Morphological (P wave) feature. The Morphological (QRS complex) used by three studies which are Islam and Alajlan 2014, Li and Li 2014, Abo-Zahhad, Ahmed et al. 2014. The ECG (P wave) and ECG (T wave) features also used in three studies which are Nomura, Ishikawa et al. 2014, Ramli, Hooi et al. 
2016, Pathoumvanh, Airphaiboon et al. 2014. Also, the feature ECG (QRS complex) used in three studies which are Islam and Alajlan 2014, Ramli, Hooi et al. 2016, Pathoumvanh, Airphaiboon et al. 2014. Thus, the Morphological (QRS complex), ECG (P wave), ECG (T wave) and ECG (QRS complex) features are desirable for making different templates between individuals with more accurate result. Each of ECG (chaos indicator), ECG (Autocorrelation (AC)), and ECG (timebased or fiducial-based) feature are just used in one studies. Hence, these features may not be proper feature for heartbeat signals.

\subsection{Biometric Template in Database}

In this step, the biometric features of individuals are stored in database. Then the stored data is used for comparison with the inputted data from verification phase to find matches for decision making [1].

\subsection{Classification}

A crucial method to identify and classify arrhythmias is the classification of heartbeats. The extracted features are compared with the feature which is stored in database [1]. In other words, classification is used to classify inputted data in different set of classes that would be easy to compare with stored data [2]. There are different methods for classification. Some of them are used in the related work studies which we reviewed as Neural Neighbor (NN), SVM, Fuzzy Logic, Euclidean Distance and Neural Network.

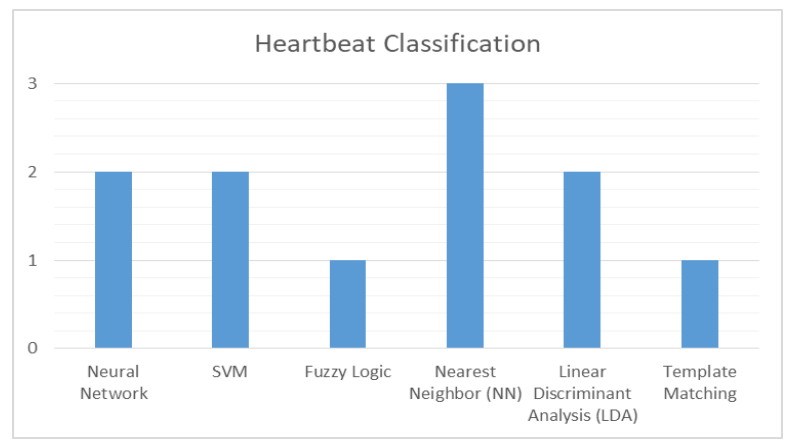

Fig. 3. Heart beat classification comparison.

As show in the Fig. 3, the chart is a about usage of different types of classifications in the recent related work studies which we have reviewed. The Nearest Neighbor (NN) classification is mostly used in the related work studies. Therefore, the most reliable classification is Nearest Neighbor (NN) because it is used in three related work studies among nine and has the highest accuracy in respect to others classifications in the chart.

\subsection{Decision}

The last step of heartbeat biometric system is decision which shows the result of inputted data for verification to YES or NO. In this step, the heartbeat biometric system compares the information which are collected after Data Acquisition, Pre-Processing, Segmentation, Feature Extraction, and classification steps with data in storage to make the final decision. Then the system will accept or deny individual [18].

\section{Evaluation of Biometrics Scheme}

For a particular biometric system to be evaluated, three important error rates need to be taken into consideration, namely: False Rejection Rate (FRR), False Acceptance Rate (FAR), Equal Error Rate (EER) and accuracy. Pattern classifier output is sensitive to many factors, including algorithm choice, amount of training data and the chosen features in the feature vector. These factors will have an effect on the performance metrics computed for each classifier [19]. 
The different types of measures to be considered when evaluating any pattern classifier are shown in Table I. It shows all the possible results in a two-class problem, with the class decisions made by the classifier in the columns, and the true, known classes in the rows. The diagonal from top left to bottom right shows the number of correctly classified patterns. True accept and true reject are seen when the classifier produces the same result as the known classification for the pattern. False accept and false reject are when the classifier produces the opposite result to the known classification. According to [19] several different types of error rates are commonly reported in biometrics which are listed below:

Table 1. Confusion Matrix for Two-Class Problem [19]

\begin{tabular}{|c|c|c|c|}
\hline & & \multicolumn{2}{|c|}{ Predicted Class } \\
\hline & & Positive & Negative \\
\hline \multirow{2}{*}{ 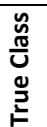 } & Positive & True Accept & False Reject \\
\hline & Negative & False Accept & True Reject \\
\hline
\end{tabular}

\subsection{False Rejection Rate (FRR)}

Equation (1) represents the number of times the system result into a false rejection (in terms of percentage). A false rejection occurs when an authorized user sample of a biometric is not matched with the stored template and then rejected by the system. Let false reject (FR) represent the number of false rejects from the classifier output and NA be the number of authorized user patterns. Then FRR is calculated using.

$$
\boldsymbol{F} \boldsymbol{R} \boldsymbol{R}=\frac{\text { Number of genuine rejects }}{\text { Number of genuine attempts }}=\frac{\mathrm{FR}}{\mathrm{NA}}
$$

\subsection{False Acceptance Rate (FAR)}

This is a statistic that represents the number of times (percentage) the system result into a false accept. This result occurs when an imposter sample biometric is matched with a stored template biometric, hence accepted by the system. Let FA be the number of false accepts and NI be the number of impostor patterns. FAR is calculated as in Equation 2.

$$
\boldsymbol{F} \boldsymbol{A R}=\frac{\text { Number of imposter accepts }}{\text { Number of imposter patterns }}=\frac{\mathrm{FA}}{\mathrm{NI}}
$$

\subsection{Equal Error Rate (EER)}

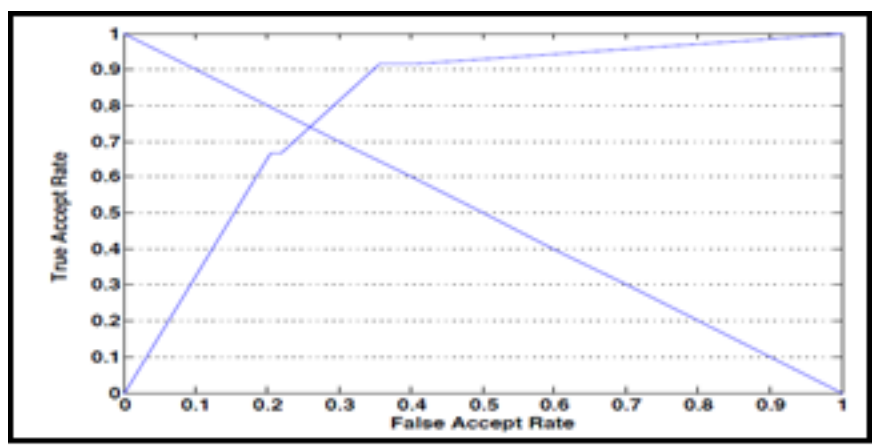

Fig. 4. The EER (25.99\%) point [19]. 
EER is the point at which the plotted curves of TAR (1-FRR) and FAR meet. As shown in Figure 4, EER can also be determined by plotting the ROC curve for the classifier, as detailed below, and determining its abscissa by plotting a diagonal line from the upper left to the lower right corners and observing where the two lines cross [20].

\subsection{Accuracy}

In as much as a confusion matrix gives all the information required, to evaluate the performance of a classification model, aggregation it would be more preferable, so that it can be easier to compare different models' performance. The confusion matrix provides the results to calculate the accuracy, it is specified as follows [21]:

$$
\text { Accuracy }=\frac{\text { Number of correct predictions }}{\text { Total number of predictions }}
$$

In most cases classification algorithms look for models that can give the highest accuracy or give the lowest error rate when applied to a training set.

\section{Related Work Result Compression}

The compression of the recent related work are listed as below; it has been done based on Feature, Classification, Number of Users, Types of devices, and Performance.

Table 2. Related Work Comparison

\begin{tabular}{|c|c|c|c|c|c|}
\hline Author & Features & Classification & No.users & Device & Performance \\
\hline $\begin{array}{c}\text { Abo-Zahhad, Ahmed } \\
\text { et al. } 2014\end{array}$ & $\begin{array}{c}\text { ECG (time-based or fiducial- } \\
\text { based) \& ECG (frequency-based) }\end{array}$ & $\begin{array}{l}\text { Neural Network } \\
\text { (NN) }\end{array}$ & 165 & ECG & EER $13.70 \%$ \\
\hline $\begin{array}{l}\text { Alajlan, Islam et al. } \\
2013\end{array}$ & Morphological ( $\mathrm{P}$ wave) & Fuzzy Logic & 112 & $\begin{array}{l}\text { Handheld ECG } \\
\text { device }\end{array}$ & EER $2.38 \%$ \\
\hline Brás and Pinho 2015 & ECG (frequency-based) & $\begin{array}{c}\text { Nearest } \\
\text { Neighbor (NN) }\end{array}$ & 52 & ECG & Accuracy $99 \%$ \\
\hline $\begin{array}{c}\text { Islam and Alajlan } \\
2014\end{array}$ & $\begin{array}{l}\text { Morphological (P-wave, QRS } \\
\text { complex and T-wave) }\end{array}$ & $\begin{array}{c}\text { Nearest } \\
\text { Neighbor (NN) }\end{array}$ & 112 & $\begin{array}{l}\text { Handheld ECG } \\
\text { device }\end{array}$ & Accuracy $70.53 \%$ \\
\hline $\mathrm{Li}$ and $\mathrm{Li} 2014$ & Morphological (P-wave) & $\begin{array}{c}\text { Nearest } \\
\text { Neighbor (NN) }\end{array}$ & 290 & ECG & EER $23.6 \%$ \\
\hline $\begin{array}{c}\text { Nomura, Ishikawa et } \\
\text { al. } 2014\end{array}$ & Chaos Indicator of ECG & $\begin{array}{l}\text { Neural Network } \\
\text { (NN) \&SVM }\end{array}$ & 20 & ECG & $\begin{array}{l}\text { Neural Network(NN) Accuracy } \\
81.0 \% \text { \& SVM Accuracy } 86.5 \%\end{array}$ \\
\hline $\begin{array}{l}\text { Pathoumvanh, } \\
\text { Airphaiboon } \text { et al. } \\
2014\end{array}$ & $\begin{array}{c}\text { ECG (P-wave, QRS complex and } \\
\text { T-wave) }\end{array}$ & $\begin{array}{c}\text { Euclidean } \\
\text { distance }\end{array}$ & 50 & ECG & Accuracy $97 \%$ \\
\hline $\begin{array}{l}\text { Pouryayevali, Wahabi } \\
\text { et al. } 2014\end{array}$ & Autocorrelation (AC) of ECG & $\begin{array}{l}\text { Euclidean } \\
\text { distance }\end{array}$ & 1020 & ECG & EER 5.0\% \\
\hline $\begin{array}{l}\text { Ramli, Hooi et al. } \\
2016\end{array}$ & $\begin{array}{c}\text { ECG (P-wave, QRS complex and } \\
\text { T-wave) }\end{array}$ & SVM & 50 & $\begin{array}{c}\text { Wearable Bracelet } \\
\text { ECG device }\end{array}$ & EER $2.0069 \%$ \\
\hline
\end{tabular}

\section{Conclusion}

In this paper, we dealt with the overall study of heartbeat biometric identification systems to reveal the ECG waveforms standards. The review recognized different methods of measuring of the heartbeat signals due to the comparing several ECG segments based on the accuracy result. Therefore, the heartbeat biometric feature, classification, number of experimented participant and the signal detector device are considered to validate the authentication layers of signals. Hence, all considered to improve the acceptance rate of signals with filtering and analyzing capability of researcher methods. Promising results are observed with the level of accuracy at highest level and lowest level EER as achieved. In result, our study acquired the last validate and straightforward of heartbeat authentication procedures with presenting of compression and analyzing efficiency of heartbeat records. 


\section{Future Works}

Our study based on the review of research papers considered the status of heartbeat biometric authentication systems focused on the biometric features traits. Future direction in our research shows that the Morphological (P wave) feature could be the most significant feature with highest rank, the ECG (P wave) and ECG (T wave) are ranked after. The Neural Network (NN) is selected as the most reliable classification with the highest performance accuracy, hence, the number of participants or users should be considered highly with respect to the number of samples. Therefore, the heartbeat authentication methods mechanism need to be experimented in the realistic circumstances. To be added, the wearable bracelet ECG device as a favorite ECG signal detector could be chosen a major research tool with Android interface software. In the result, for future explore to propose a system for heartbeat biometric authentication all the mentioned points should be considered with their mechanism to have the best efficiency regarding identifying the correct and accrued result from the subject heartbeat signals.

\section{Acknowledgments}

The authors would like to thank American University of Afghanistan (AUAF) for their educational and financial support. This work is conducted at the AUAF Information Technology \& Computer Science department and funded by American University of Afghanistan.

\section{References}

[1] Abo-Zahhad, M., Ahmed, S. M., \& Abbas, S. N. (2014). Biometric authentication based on PCG and ECG signals: present status and future directions. Signal, Image and Video Processing, 8(4), 739-751.

[2] Alariki, A. A., Manaf, A. A., \& Mousavi, S. M. (2016). Features extraction scheme for behavioural biometric authentication in touchscreen mobile devices. International Journal of Applied Engineering Research, 11(18), 9331-9344.

[3] Council, N. R., \& Committee, W. B. (2010). Biometric Recognition: Challenges and Opportunities. National Academies Press.

[4] Unar, J., Seng, W. C., \& Abbasi, A. (2014). A review of biometric technology along with trends and prospects. Pattern Recognition, 47(8), 2673-2688.

[5] Pathoumvanh, S., Airphaiboon, S., \& Hamamoto, K. (2014). Robustness study of ECG biometric identification in heart rate variability conditions. IEEJ Transactions on Electrical and Electronic Engineering, 9(3), 294-301.

[6] Jain, A., \& Pankanti, S. (2001). Biometrics systems: Anatomy of performance. IEICE Transactions on Information and Systems, 84(7), 788-799.

[7] Gamboa, H., \& Fred, A. (2004). A behavioral biometric system based on human-computer interaction. Proceedings of SPIE, 5404, 381-392.

[8] Jomaa, R. M., Islam, M. S., \& Mathkour, H. (2015). Enhancing the information content of fingerprint biometrics with heartbeat signal. Proceedings of the World Symposium on Computer Networks and Information Security (WSCNIS) (pp. 1-5).

[9] Ramli, D., Hooi, M., \& Chee, K. (2016). Development of heartbeat detection kit for biometric authentication system. Procedia Computer Science, 96, 305-314.

[10] Alajlan, N., Islam, M. S., \& Ammour, N. (2013). Fusion of fingerprint and heartbeat biometrics using fuzzy adaptive genetic algorithm. Proceedings of the 2013 World Congress on Internet Security (WorldCIS) (pp. 76-81).

[11] Brás, S., \& Pinho, A. J. (2015). ECG biometric identification: A compression based approach. Proceedings of the 37th Annual International Conference of the IEEE Engineering in Medicine and Biology Society 
(EMBC) (5838-5841).

[12] Islam, M. S., \& Alajlan, N. (2014). Model-based alignment of heartbeat morphology for enhancing human recognition capability. The Computer Journal, 58(10), 2622-2635.

[13] Li, M., \& Li, X. (2014). Verification based ECG biometrics with cardiac irregular conditions using heartbeat level and segment level information fusion. Proceedings of the 2014 IEEE International Conference on Acoustics, Speech and Signal Processing (ICASSP) (3769-3773).

[14] Nomura, R., Ishikawa, Y., Umeda, T., Takata, M., Kamo, H., \& Joe, K. (2014). Biometrics authentication based on chaotic heartbeat waveform. Proceedings of the 7th Biomedical Engineering International Conference (BMEiCON) (pp. 1-5).

[15] Pouryayevali, S., Wahabi, S., Hari, S., \& Hatzinakos, D. (2014). On establishing evaluation standards for ECG biometrics. Proceedings of IEEE International Conference on Acoustics, Speech and Signal Processing (ICASSP) (pp. 3774-3778).

[16] Islam, M. S., \& Alajlan, N. (2017). Biometric template extraction from a heartbeat signal captured from fingers. Multimedia Tools and Applications, 1-25.

[17] Ye, C., Kumar, B. V., \& Coimbra, M. T. (2012). Heartbeat classification using morphological and dynamic features of ECG signals. IEEE Transactions on Biomedical Engineering, 59(10), 2930-2941.

[18] Sanjana, G. Human Authentication Based on ECG Imaging.

[19] Crawford, H. A. (2012). A Framework for Continuous, Transparent Authentication on Mobile Devices. University of Glasgow.

[20] Clarke, N. L., Furnell, S. M., \& Reynolds, P. L. (2002). Biometric authentication for mobile devices. Proceedings of the 3rd Australian Information Warfare and Security Conference (pp. 61-69).

[21] Saevanee, H., Clarke, N., Furnell, S., \& Biscione, V. (2015). Continuous user authentication using multimodal biometrics. Computers \& Security, 53, 234-246.

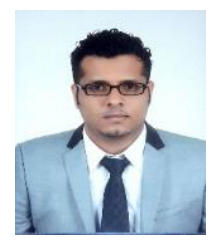

Ala Abdulhakim Abdulaziz is currently an assistant professor at the department of Information Technology and Computer Science at American University of Afghanistan. He received his $\mathrm{PhD}$ and MITS in computer science from the University of Technology Malaysia, Kuala Lumpur, Malaysia. He obtained his B.Sc in computer science from Multimedia University. His research interests include a variety of topics in the areas of machine learning, artificial intelligence, biometrics technology, etc.

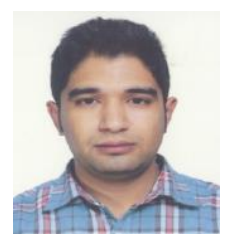

Sayed Mahmoud Alavy received his bachelor degree in computer science and information technology in 2017 from American University of Afghanistan. He has more than 8 years of experience in information technology field.

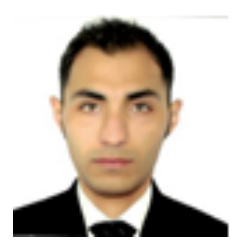

Mohammad Reza Yousufi received his bachelor degree in computer science and information technology in 2017 from American University of Afghanistan. He has more than 8 years of experience in information technology field.

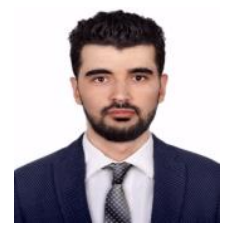

Mohammad Tareq Aziz, senior student of Computer Science and Information Technology Department at American University of Afghanistan. He has 13 years of experience in information technology field. 
Christine Murray received her Ph.D from University of Texas at Arlington in 2005. She is assistant professor of English at American University of Afghanistan, teaching technical writing, argument, and literature. 